\title{
BODIES IN FLOWING PLASMAS: SPACECRAFT MEASUREMENTS
}

\author{
Uri Samir \\ Space Physics Research Laboratory, The University of \\ Michigan, Ann Arbor, Michigan 48109, USA; and Department \\ of Geophysics and Planetary Sciences, Tel Aviv University, \\ Israel
}

ABSTRACT

Results from in-situ measurements relevant to the interaction of bodies in flowing plasmas are reviewed. A brief discussion of the interaction in the general context of SPACE PIASMA PHYSICS, including possible applications to solar-system plasmas is given. The mode of experimentation in the Shuttle/Spacelab era is also mentioned.

\section{BACKGROUND COMMENTS}

Understanding the phenomena involved in the interaction between highly rarefied plasmas and rapidly moving bodies is fundamental to Solar-System Plasma Physics and to Space Plasma Physics at large. The study of flow interactions between planets and some of their natural satellites with the solar wind and planetary magnetospheres is now over a decade old. However, only recently [1] have attempts been made to seek a "unifled-theory" for body-plasma interactions. Body-plasma interaction in the solar system can be: (1) between the solar wind and a planetary magnetic field, (2) between the solar wind and a planetary ionosphere-atmosphere and (3) between a planetary environment and/or solar wind with the surface of the body. Hence, the structure of the flow fields ahead of and behind the body have specific characteristics for each case. A particular case of both scientific and practical interest is that of a spacecraft orbiting the earth. Here, the interaction takes place in a supersonic and sub-Alfvenic flow regime, which may suggest application to the motion of natural satellites orbiting their parent planets in the outer solar system. Although the motion of an artificial satellite in a planetary ionosphere can serve as a 'model' for some typical interactions in the solar system, the entire complex of phenomena involved in such interactions is not yet well-known. In the past, the interaction between satellites and the terrestrial ionosphere was of interest because of (1) its relevance to the reliability of in-situ measurements performed by current-collection devices $(e . g \cdot[2,3]$, and (2) the effects such interactions have on the scattering of electromagnetic waves [4] from satellite trails. Many theoretical papers dealing with different aspects of the interaction were published $[2,4-12]$. Various aspects of the interaction of earth-orbiting satellites were investigated experimentally via in-situ observations [13-17] and via laboratory simulation experiments $[3,18-20]$. However, despite the above efforts, the information currently available from both theoretical and experimental work is fragmentary and insufficient for an adequate exami- 
nation of the physical processes involved in such interactions. Some of the processes involved in the above interactions are of interest to the physics of rarefied plasmas in general, and are applicable to specific cases of body-plasma interactions in the solar system. The in-situ information available for analysis, is limited and fragmentary due to: (1) the fact that most diagnostic probes were flush-mounted on the surfaces of the satellites and/or on relatively short boons confining the measurements to the very near vicinity of the satellite's surface, (2) the fact that most in-situ data are obtained as by-products of experiments designed for geophysical purposes, hence the data results from non-optimal space experiments and (3) the fact that experiments could not be performed in a CONTROLLED manner.

With the advent of the Shuttle/Spacelab, it becomes possible to use the terrestrial environment as a 'working' rarefied plasma and the Spacelab/orbiter as a 'nearearth' laboratory suitable for performing body-plasma interaction studies in a wide scientific context. The approach of using the Spacelab/Orbiter as an ACTIVE laboratory where CONTROLLED experiments can be performed is a new experimental concept, which combines the experience and methods used in space research together with the practices and methods of experimentation used in plasma-chamber research. A wide spectrum of problems can be studied via the utilization of the Spacelab/Orbiter and its wide range of capabilities. Among problems of fundamental interest to space plasma physics are: (1) the nature of collisionless super sonic and sub-Alfvenic flows including body-plasma interactions in this regime (applicable for example, to the motion of natural satellites such as Io and Titan in the environment of their parent planets), (2) parametric conditions for the applicability of magnetohydrodynamics and kinetic approaches in dealing with bodyplasma interactions in the solar system. We expect the next generation of experiments relevant to BODY-PLASMA interactions in its widest context to be performed on board large space platforms. Such in-situ studies could be complemented by laboratory-simulation experiments performed in plasma chambers, thereby extending the range of plasma flow regimes to be studied. This introduces the question of 'scaling'. Traditionally, when 'simulation' of cosmic, astrophysics or planetary phenomena was attempted, the efforts focused on the simulation of 'entire systems' or 'configurations' in space. In attempting the latter, the effort appeared sometimes useless. However, as discussed by Falthammar [21]; Block [22]; Podgorny and Andrijanov [23] and others, it is possible to use the principle of qualitative scaling' and achieve 'process simulation'. Experimental work, both in-situ on board large space platforms and via laboratory-simulation can utilize this principle and achieve results of fundamental significance to Space Plasma Physics. In sum, the next generation of well-conceived experiments in the general area of BODY-PLASMA interactions should proceed along two avenues: (1) CONTROLLED and ACTIVE in-situ measurements performed on board the Spacelab/Orbiter or any other large space platform and (2) laboratory-simulation measurements in plasma chambers. For each mode of experimentation the principle of 'qualitative scaling' could be used, thereby extending the range of scientific return.

\section{IN-SITU MEASUREMENTS}

\section{1: General Comments}

Most of the information available at the present from in-situ measurements is relevant to the interaction between a spacecraft and the terrestrial ionosphere. This information stems from selected samples of measurements made by planar, spherical and cylindrical Langmuir probes, retarding potential analyzers and ion mass spectrometers mounted on board ionospheric satellites. During the $1960^{\prime} \mathrm{s}$, measurements from the U.K./Ariel 1, Explorer 31 and the Gemini-Agena 10 were used, and during the 1970's measurements from the Explorer 31, Atmosphere Explorer C and $\mathrm{E}$ and the USAF/S3-2 satellites were used. For all cases, measurements of: 
$I_{e}, N_{e}, I_{+}, N_{+}, T_{e}, T_{+}, \phi_{s}$ and $M_{+}$(where $I$ and $N$ represent current and number densities for electrons and ions respectively, $\mathrm{T}=$ temperature, $\phi_{\mathrm{S}}=$ satellite potential with respect to the ambient plasma and $\mathcal{M}_{4}=$ ionic composition) were sought. In most cases (e.g. Samir [24], the probes were flush-mounted on the surfaces of the satellite except for the cylindrical electrostatic probes (Brace et $\mathrm{al}$, [25] on board the Atmosphere Explorer $C(\Lambda E-C)$ and the guarded planar probe which was mounted on a $5 \mathrm{~m}$ long boom on board the Ariel 1 satellite. The available information is mostly relevant $t_{0}: I=f\left(r=R_{0}, \sim 2 R_{O}, 5 R_{0}\right.$; $\left.\theta\right)$ where: $\mathrm{R}_{\mathrm{O}}=$ the 'effective radius' of the satellite and $\theta=$ the angle of attack. Hence, an a-priori limitation is imposed on the measurements namely, being confined to the nearest vicinity of the satellite surface. Far regions, namely, regions for which $\mathrm{d}>\mathrm{S} \mathrm{R}_{\mathrm{o}}$ (where: $\mathrm{d}=$ distance from satellite center, $\mathrm{S}=$ ionic Mach number) ahead and behind the satellites could not have been studied via direct measurements. Figure 1 is a schematic drawing showing satellite geometry and probe location. During the $1960^{\prime}$ s most observations were exploratory and phenomenological [13] and showed the existence of a wake depleted of ions and electrons with a net negative charge. The early results from the Explorer 31 (late 1960's and early 1970's) confirmed the gross features observed earlier.
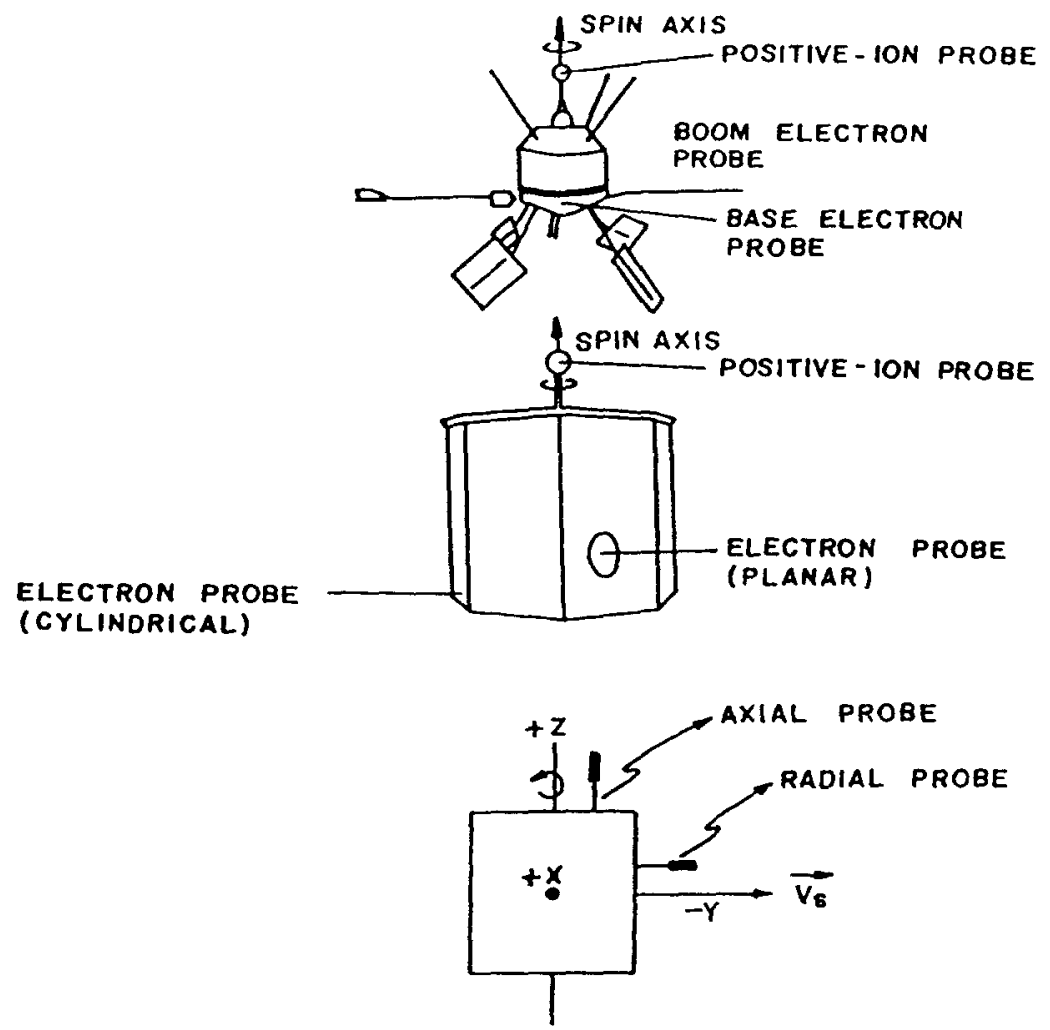

Fig. 1. A schematic diagram showing the Ariel 1, Explorer 31 and AE-C satellites including the location of some probes. 
During the 1970 's, attempts were made to compare theoretical wake models with relevant in-situ measurements, e.g. $[4,6,14,26-29]$. The main deficiency of early studies is that no systematic parametric investigations were performed. Hence, the major effort during the 1970's focused on the latter (e.g. [15, 16].

\subsection{Discussion of some significant results.}

A. Electron ion and potential distribution in the near wake of an ionospheric satellite.

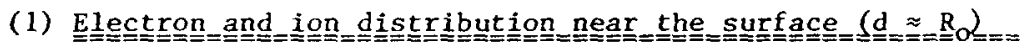

The distribution of electrons, ions and local potential around ionospheric satelittes was studied via measurements of probes mounted on the surfaces of the: Arie1 1, Explorer 31, $\mathrm{AE}-\mathrm{C}$ and $\mathrm{S} 3-2$ satel1ites $[15,24,30-33]$.

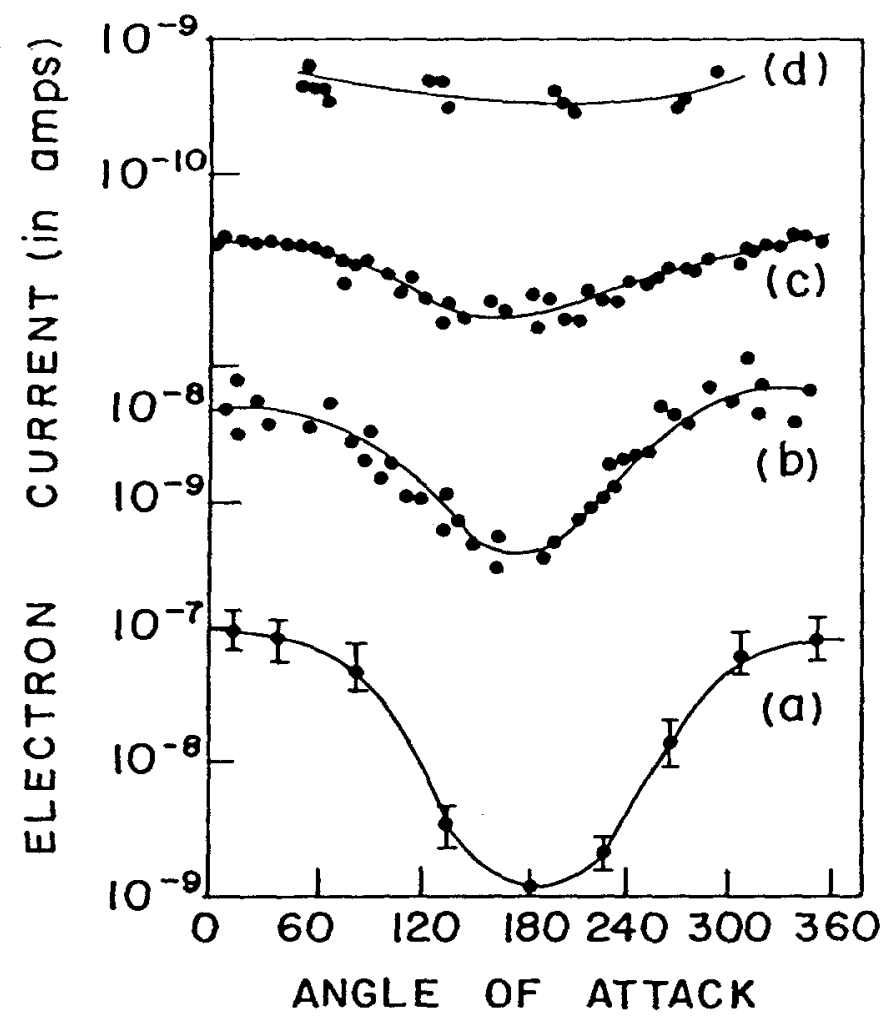

Fig. 2. Variation of $I_{e}=f(\theta)$ at the surface of an ionospheric satellite in the altitude ranges: (a) $430-600 \mathrm{~km}$, (b) $620-910 \mathrm{~km}$, (e) $1175-1685 \mathrm{~km}$ and (d) 2060-2280 km (After: Samir and Willmore, 1965; Samir and Wrenn, 1969).

Figure 2 shows an average picture of $I_{e}=f(\theta)$ at $d \approx R_{0}$ based on measurements from the Ariel 1 and the Explorer 31 satellites. As seen from the figure the ratio $\alpha=\mid I_{e}$ (wake)/I (ambient) $\mid$ varies in the range $10^{-2}$ to $\approx 1$ with $\alpha \rightarrow 1$ for the higher altitudes. Hence, $\alpha \sim 10^{-2}$ depicts the amount of electron 
depletion in the wake for a plasma whose major ionic constituent is $\left|0^{+}\right|$and $\alpha \leqslant 1$ depicts the amount of electron depletion in the wake for a plasma whose major ionic constituent is $\left|H^{+}\right|$. Another finding was that no build-up of electrons exists at any altitude ahead of the satellite. Figure 3 shows the variation of normalized ion and electron currents with the angle of attack $(\theta)$ in

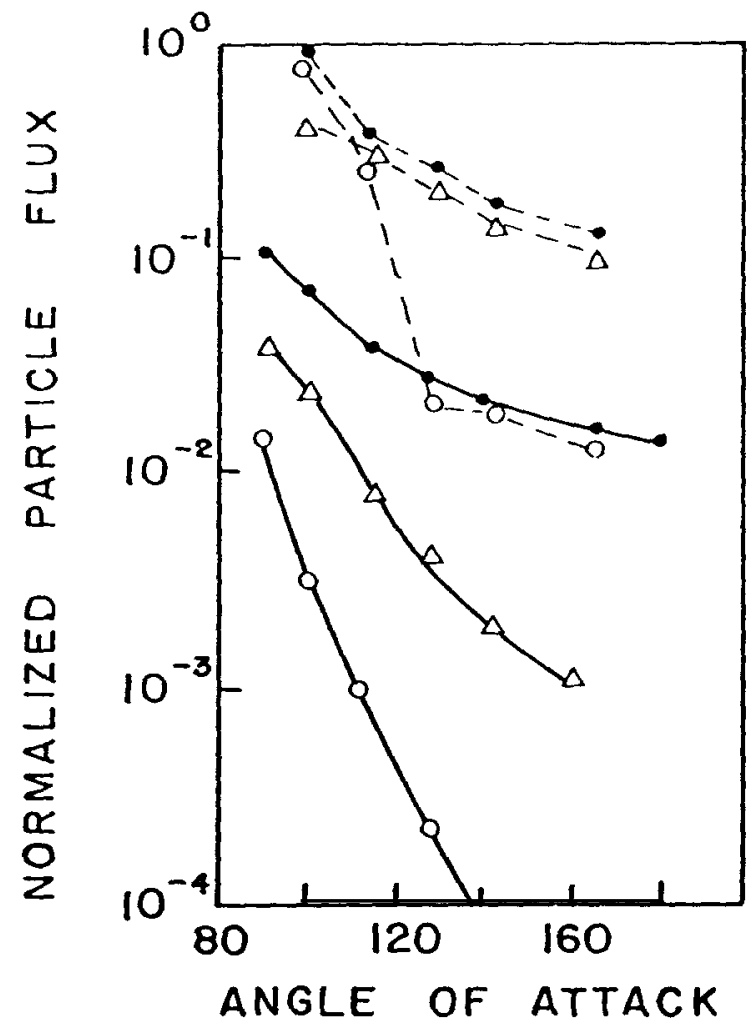

Fig. 3. Variation of normalized ion currents $I_{+}(\theta) / I_{+}\left(\theta=0^{0}\right)$ (solid lines) and electron currents (dashed lines) in the wake of the Explorer 31 satellite in the altitude ranges: 520-570 km (o); $600-900 \mathrm{~km}(\Delta)$ and $700-930 \mathrm{~km}(\cdot)$. After Samir et al, 1973.

the wake of the Explorer 31 satellite, in the altitude ranges: $520-570 \mathrm{~km}$, $600-900 \mathrm{~km}$ and $700-930 \mathrm{~km}$. The solid lines represent the ion current variation and the dashed lines represent the electron current variation $|24|$. The result shows quantitatively the difference between $I_{+}$and $I_{e}$ and as expected the difference increases as we proceed further into the wake region (i,e. larger angles of attack). The plasma parameters referring to each of the altitude ranges are: (1) $\mathrm{R}_{\mathrm{D}}=20, \mathrm{~S}_{\mathrm{AV}}=4.8, \phi_{\mathrm{N}}=-4.6, \mathrm{M}_{4}=12,\left|\mathrm{~T}_{\mathrm{e}} / \mathrm{T}_{+}\right|=1.09$ for the altitude range $700-900 \mathrm{~km},(2) \mathrm{R}_{\mathrm{D}}=19, \mathrm{~S}_{\mathrm{AV}}=3.9, \phi_{\mathrm{N}}=-3.1, \mathrm{M}_{+}=12.6,\left|\mathrm{~T}_{\mathrm{e}} / \mathrm{T}_{\mathrm{t}}\right|=1.06$ for the altitude range $600-900 \mathrm{~km}$, and (3) $\mathrm{R}_{\mathrm{D}}=56, \mathrm{~S}_{\mathrm{AV}}=5 / 6, \phi_{\mathrm{N}}=-3 . \overline{6}, \mathrm{~K}_{4}=16.0$, $\left|\mathrm{T}_{\mathrm{e}} / \mathrm{T}_{+}\right|=1.28$ for the altitude range $520-570 \mathrm{~km}$. It should be noted that the results shown in Figure 3 are from measurements of a retarding potential analyzer (R.P.A) whereas the results shown in Figure 2 are from guarded planar probes whose method (a.c. probe) differs from that used by the R.P.A. Recently, measurements from planar ion probes mounted on the surface of the $S 3-2$ satellite were used to examine the variation of $B=\mid I_{+}$(wake)/ $L_{+}$(ambient) $\mid$with altitude. Preliminary results show the amount of ion depletion in the wake of the $53-2$ satellite to be larger compared with that obtained for the Explorer 31. This can be understood in terms of the variation of ion current in the wake with the parameter 
$R_{D}\left(=R_{0} / \lambda_{0}\right) . \quad$ This study is in progress.

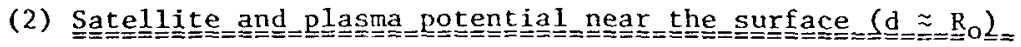

The angular variation of the equilibrium potential ( $\phi_{S}$ ) (or: 'space potential') of an ionospheric satellite with ionospheric properties was studied using measurements from the Ariel l, the Explorer 31 and the AE-C satellite [15,31,33]. It was found that the difference between space potential values and environmental plasma potential do not exceed $(0.5-0.7)$ volts. Measurements from the cylindrical electrostatic probe (CEP) on board the AE-C satellite and measurements from a planar guarded (a.c.) probe on board the Explorer 31 satellite were used to examine the variation of $\gamma \equiv \mid \phi_{s}$ (measured) $/ \phi_{s}$ (computed) $\mid=f\left(T_{e}\right)$ for: $950^{\circ} \mathrm{K}<\mathrm{T}_{\mathrm{e}}<3300^{\circ} \mathrm{K}$, where $\mid \phi_{\mathrm{S}}$ (computed) $\mid$ is the commonly used simple expression. It was found that $\gamma>1$. For the $\mathrm{AE}-\mathrm{C}$ results where: $950^{\circ} \mathrm{K}<\mathrm{T}_{\mathrm{e}}<1050^{\circ}$ we have $\gamma \approx 2.5$ whereas for the Explorer 31 results where: $1400^{\circ} \mathrm{K}<\mathrm{T}_{\mathrm{e}}<3300^{\circ} \mathrm{K}, \mathrm{a}$ dependence of $\gamma=f\left(T_{e}\right)$ is suggestive with $\gamma \rightarrow 1$ for higher values of $T_{e}$. The result is shown in Figure 4. Further details on this study are given in Samir et a1. [15].

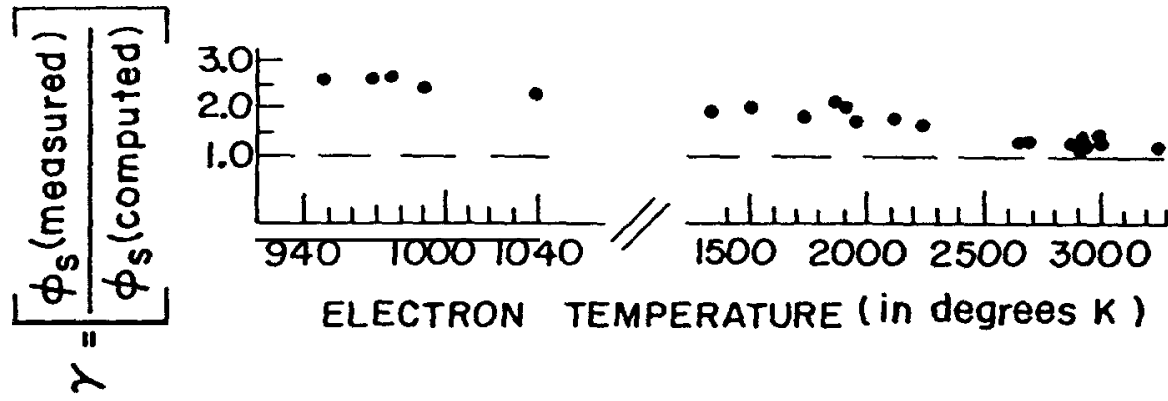

Fig. 4. Variation of the ratio $\gamma=\mid \phi_{s}$ (measured) $/ \phi_{s}$ (computed) with ambient electron temperature $\left(\mathrm{T}_{\mathrm{e}}\right.$ in $\mathrm{K}$ ). (Left) Atmosphere Explorer $\mathrm{C}$ ( $\mathrm{AE}-\mathrm{C}$ ) data. (Right) Explorer 31 data. After Samir et al, 1979.

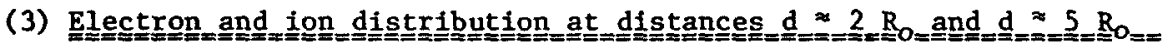
Miller [34] studied the $I_{e}=f(\theta)$ at a distance $d \approx 2$ Ro from the center of the satellite, using measurements from the cylindrical Langmuir probe on board the Explorer 31 satellite (see Figure 1). He indicated the possible existence of an enhancement in $I_{e}$ at $\theta=120$. Examination of Ariel 1 data [30] shows the possibility of a similar enhancement in $I_{+}$. However, since the sample of data used is relatively sma11, the latter claim needs further confirmation. If this result is confirmed, it may be used to assess the role played by electric fields in the near wake zone [4]. Figure 5 shows the angular variation of the normalized electron current (i.e. $\left|I_{e} / I_{0}\right|=f(\theta)$ ) behind: (a) the main body of the Ariel 1 satellite and (b) the spherical ion probe as measured by the planar probe mounted on the boom, (see Figure 1) at a distance of $d \sim 5 R_{0}$ from the center of the satellite. The main conclusions from the results shown in Figure 5 are that similar wake structures can be obtained from bodies which differ in their $R_{D}$ and $\phi_{\mathrm{N}}$ parameters. That both wakes indicate an enhancement of current on the wake axis and show the wake to be wider than the 'geometrical wake'width. It should be noticed that $R_{0}$ (satellite/ $R_{0}$ (probe) $\approx 6$ and a similar value existed for $\mid \phi$ (probe)/申(satelifte)|. The ionic Mach number for the sample of data used in the above study was $S \approx 4$. Further details on this study are given in [32]. 


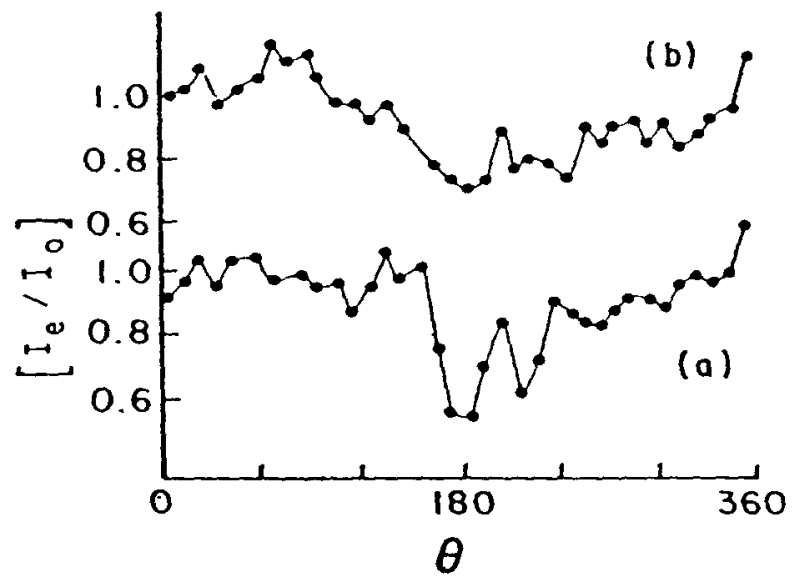

Fig. 5. Variation of notmalized electron current $I_{e} / I_{0}$ with angle of attack ( $\theta)$ behind: (a) the main body of the Ariel 1 satellite and (b) the spherical ion probe. Arter Henderson and Samir, 1967.

A useful quantitative result obtained by comparing the average value $\left(I_{e} / I_{0}\right), a t$ $d \approx 5 R_{0}$ with the average value of $\left(I_{e} / I_{0}\right)$ at $d \approx R_{0}$ shows $I_{e} / I_{0}$ at $d \approx 5 R_{0}$ to $\left(I_{\mathrm{e}} / I_{\mathrm{o}}\right)$ at $\mathrm{d} \approx 1 \mathrm{R}_{\mathrm{o}}$ to be about 50, for a plasma with $\mathrm{R}_{\mathrm{D}} \approx 10$ and $\mathrm{S} \approx 3.75$. This result was also used for theory-experiment comparison $[4,6,28]$. Studies relevant to the distribution of ions and electrons around an ionospheric satellite and particularly the distribution in the wake were also made by: Oya [35], Weil and Yorks [36], and by Medved, Troy and Samir $|37,38|$. The work by Medved et a1. was done using the Agena-Gemini 10 system. The Agena was a large body compared with ionospheric satellites like Ariel and Explorer and indeed the normalized ion current $\beta$ at $\theta=150$ - 180 showed this ratio to be several orders of magnitude smaller than the larger values obtained for the Explorer 31 (see Figure 3).

Furthermore, during an axial maneuver performed by the Gemini capsule relative to the Agena, an approximate $\left(I_{e} / I_{0}\right)$ and $\left(I_{+} / I_{0}\right)$ axial profile was obtained, indicating an enhancement in current at a distance of about $z \approx S R_{0}$ in the wake. This result is in general accord with results shown in Figure 5. The implication of this result to interpreting wake characteristics in terms of a 'two stream' flow model is given in $[20]$.

\section{B. About an electron temperature enhancement in the wake,}

A study of the angular distribution of electron temperature around a satellite at $\mathrm{d} \approx \mathrm{R}_{\mathrm{O}}$ indicated the electron temperature in the wake to exceed that of the ambient electron temperature. Figure 6 shows the variation of electron temperacure with altitude for two angle of attack ranges: (a) $0^{\circ} \leq \theta \leq 60^{\circ}$ and (b) $150^{\circ} \leq \theta \leq 180^{\circ}$ as measured by a planar probe flush-mounted on the surface of the Explorer 31 satellite [39]. A similar study, using measurements from an R.P.A. mounted on the surface of the same satellite [40] showed a similar result. Electron temperature measurements made by a similar probe mounted on the boom on the Ariel 1 satellite namely, at a distance $d \approx 5 R_{0}$ from the satellite center (Fig.1) did not show any such temperature enhancement. The $T_{e}=f(\theta)$ study was extended and possible effects of the geomagnetic field on electron temperature were examined (e.g. $[39,40]$. The outcome of the study shows $T_{e}=f(\theta)$ to be more significant than $T_{e}=E\left(B_{E}\right)$, where: $B_{E}=$ geomagnetic field. Moreover, it has been shown that the magnitude of the enhancement does not depend on the average ion mass, although the electron density depletion in the wake is strongly correlated with ionic mass [40]. It should be noticed further that the Gemini-Agena 10 
experiment also indicated an electron temperature enhancement in the wake [38]. The cause of the temperature enhancement is not yet clear. Since we know $[2]$ that behind the satellite a negative potential well exists, it is possible that in this well, wave-particle interactions take place and apply an energy filtering mechanism to the electrons that leave it. The resulting population close to the satellite can then have an effective temperature higher than that of the ambient thermal electrons. Alternatively, it is possible to infer the existence of heating

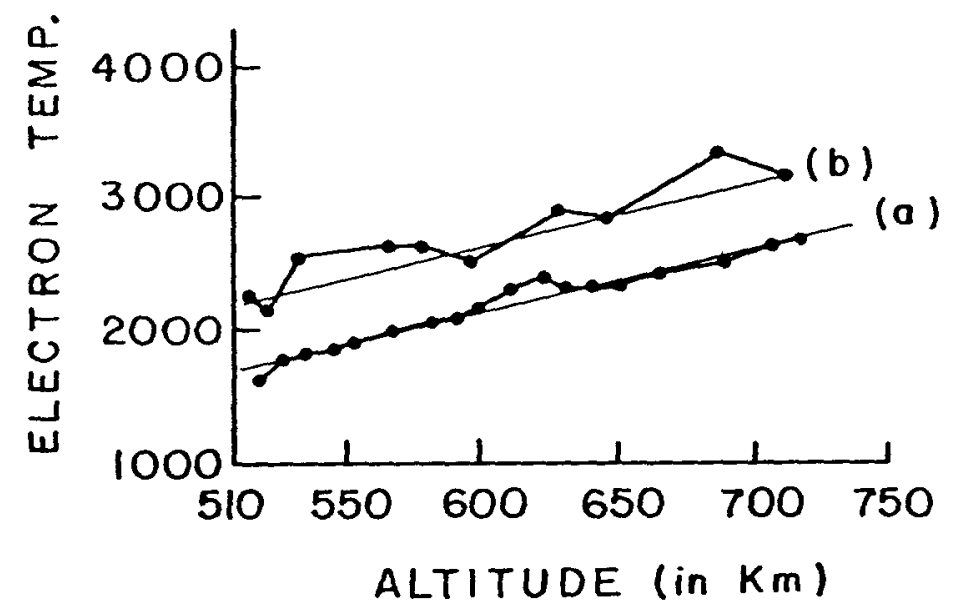

Fig. 6. Variation of electron temperature with altitude for two angles of attack $(\theta)$ ranges: (a) $0^{\circ} \leq \theta \leq 60^{\circ}$, (b) $150^{\circ} \leq \theta \leq 180^{\circ}$. Af ter: Samir and Wrenn, 1972.

mechanisms in the wake region due to stream interactions and/or plasma instabilities correlated with plasma oscillations in the near wake [39]. The existence of the latter was suggested by Samir and willmore $[30]$, and discussed in $[4,6]$. IIliano and Storey [41] suggested that the $T_{e}$ (wake) enhancement is 'apparent' rather than real, based on the possible usage of a truncated Maxwellian produced by a shift in the space charge potential. As discussed by Troy et al [40], this suggestion is unlikely. Gurevich et al, [42] discuss the existence of ion acceleration mechanisms upon the expansion of a rarefied plasma into a vacuum. Such mechanisms may be relevant to the above discussion.

C. Evidence for the existence of plasma oscillations in the near wake zone.

Measurements from the Ariel 1 satellite have indicated the possible existence of plasma oscillations near the steep density gradients at the wake boundary. The oscillations refer to temporal variations of the plasma in the satellite's frame of reference and their frequency has a component of about $3 \mathrm{kc} / \mathrm{sec}$. This finding is in accord with theoretical calculation $[2,4,6]$, in particular with the plasma instability in the wake of a moving body which generates oscillations with frequencies of the order of $5 \mathrm{kc} / \mathrm{sec}$ for a plasma with $\mathrm{N}=10^{5} \mathrm{~cm}^{-3} \mathrm{M}_{+}=16$ similar to the experimental conditions of $N_{0} \approx 10^{4}-10^{5}$ and $M_{+}=[16]$ given in [30]. This result may be connected to the electron temperature enhancement discussed in the preceding section. It is expected that Spacelab/Orbiter experi- 
ments would include measurements directly ained at scarching for such plasma oscillations.

D. Alout some recent parametric studies.

Parametric studies which could contribute to a betcer understanding of the physical processes involved in BODY-PLASMA interactions seldom materialized in the past (Section 2.1). This situation was rectified somewhat by using Atmosphere Explorer data. And indeed, the analysis of measurements performed by the ensemble of instruments on board the $A E-C$ and $A E-E$ satellites contributed significantly to parametric studies. The results discussed below are a partial outcome of analyses which made use of measurements from the cylindrical electrostatic probe (CEP) (Brace et a1,) [25] and the Bennett Ion Mass Spectrometer (BIMS) (Brinton et al.,) [43]. Using measurements of ion current electron temperature ionic composition and values of space potential the parameters: $R_{D} T_{e} / T_{+} ; S ; \phi_{N}$ were computed for each of the data samples used. The angular distribution of ion current at a distance of $d \approx 0.5 \mathrm{R}_{0}$ from the satellite's surface, was investigated for the parameter ranges: $58 \leq R_{\mathrm{D}} \leq 162 ; 1.07 \leq\left|\mathrm{T}_{\mathrm{e}} / \mathrm{T}_{+}\right| \leq 1.21$; $5.93<\mathrm{S}_{\mathrm{AV}}<8.04 ; 8.4 \leq\left|\phi_{\mathrm{N}}\right| \leq 9.5$ which by far extend parametric ranges used in earlier studies. It was found that $I_{+} / I_{0}=f(\theta)$ behaves differently for different sets of parameters, particularly having different values of $R_{D}$. For example, the amount of ion depletion in the wake (i.e. $\mid I\left(\theta \approx 165^{\circ}\right) / I_{+}$(ambient) $\mid$) for the parametric conditions: $R_{D}=162.5 ;\left|T_{e} / T_{+}\right| \approx+1.1 ; S=7.7 ;\left|\phi_{N}\right| \approx 8.4$ was by at least one order of magnitude larger than the ion depletion for the parametric conditions: $\mathrm{R}_{\mathrm{D}}=83.8 ;\left|\mathrm{T}_{\mathrm{e}} / \mathrm{T}_{+}\right| \approx 1.1 ; \mathrm{S}=7.9 ;\left|\phi_{\mathrm{N}}\right|=9.3$. Moreover, it was found that $I_{+}$(wake) $7 I_{+}$(ambient) displays an exponential dependence on $R_{D}$ for 'constant' values of other relevant parameters $|15 ; 44|$. Figure 7 shows the variation of $\mid N_{+}\left(\theta=160^{\circ}\right) / N_{+}($ambient $) \mid$with $R_{D}$ for $37 \leq R_{D} \leq 247$. The exponential dependence can be written in the form: $\beta=a_{0} \exp \left(a_{1} \cdot R_{D}\right)$ where: $\beta=$ the ratio of the density in the wake to ambient density and $a_{0}=0.06 ; a_{1}=-0.009$. It should be realized that the establishment of such empirical relationships should aid in testing theoretical wake models, $i . e$. help assess the validity and range of applicability of physical assumptions used in the models. Samir and Kaufman [29] performed a partial parametric theory-experiment comparison, and found that a combined approximation which is a weighted sum of a neutral approximation for $\left|\mathrm{H}^{+}\right|$and a quasi-neutral approximation for $\left|\mathrm{O}^{+}\right|$yield better results than any of these approximations separately. Another parametric investigation [16] indicated that both electron temperature and ionic composition significantly influence the amount of ion depletion in the wake as shown in Figure 8 . In this figure the variation of $\beta$ with electron temperature for various values of $N\left(O^{+}\right) / N\left(H^{+}\right)$are shown. It should be realized that in addition to the scientific value of these parametric investigations, results can be used in the planning and design phase of CONTROLLED experiments to be performed in the future on board large space platforms. 


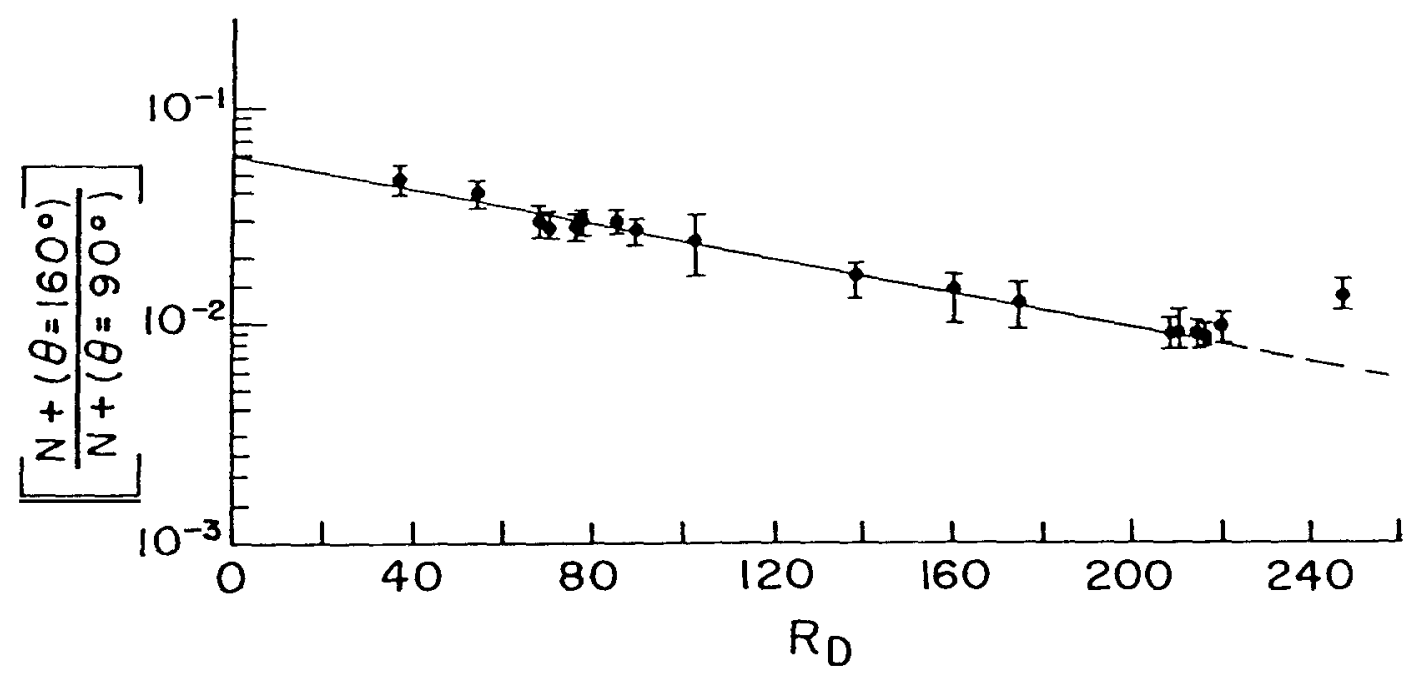

Fig. 7. Variation of $N_{+}\left(\theta=160^{\circ}\right) / N_{+}\left(\theta=90^{\circ}\right)$ with $R_{D}=R_{o} / \lambda_{D}$. After Samir et a1, 1980.

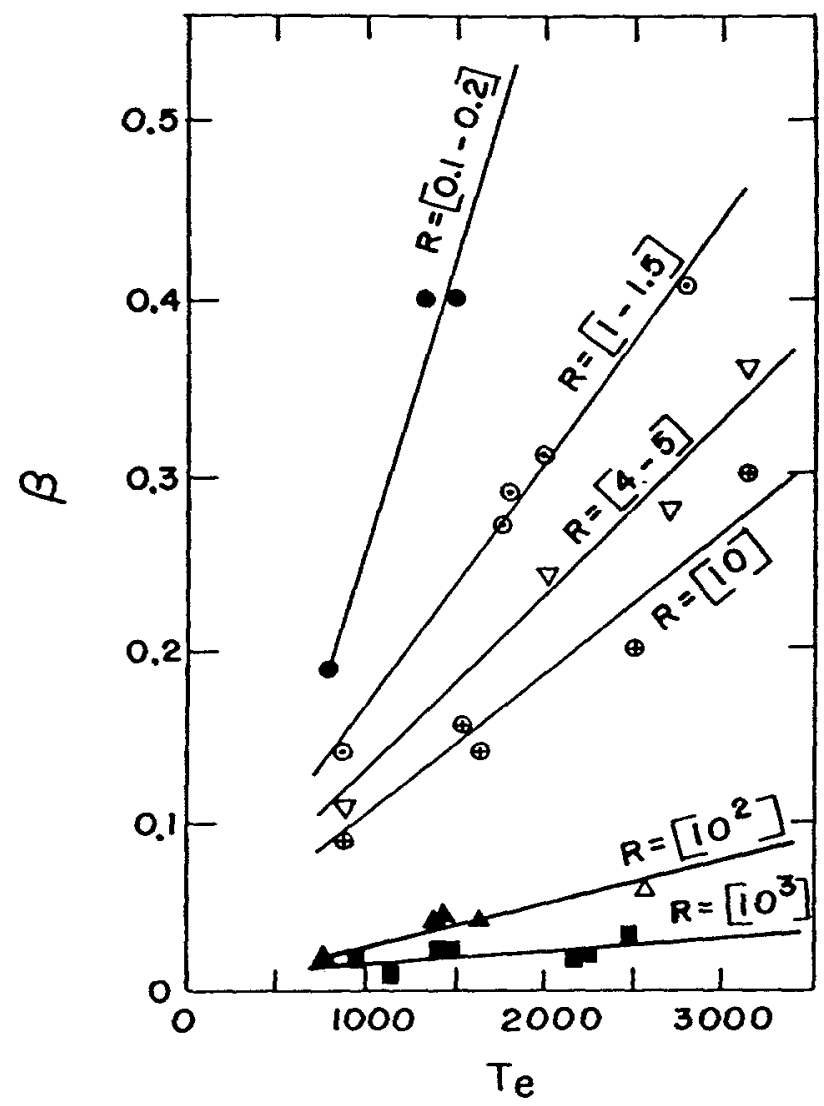

Fig. 8. Variation of $\beta=\mid I_{+}$(wake) $/ I_{+}$(ambient) $\mid$ with $T_{e}$ for various values of $\mathrm{R}=\left|\mathrm{N}\left(\mathrm{O}^{+}\right) / \mathrm{N}\left(\mathrm{H}^{+}\right)\right|$. After Samir et al 1979. 
SUMMARY

Most in-situ investigations performed during the past decade were limited to the very near surface of ionospheric satellites. An extension of the studies to the exploration of further regions would have required the use of multi-body systems or long booms. Such were not available for scientific work. It is expected that experiments to be performed on board the Spacelab/orbiter will allow the performance of we11-planned CONTROLLED experiments in the area of BODY-PLASMA interactions in its widest sense. Despite the a-priori limitations mentioned, the parametric studies already performed have contributed significantly towards a better understanding of the parametric interplay hence the acting physical processes.

\section{ACKNOWLEDGEMENT :}

The author acknowledges the interest and support of the office of Space Sciences at NASA Headquarters and the Atmospheric Division at the NSF.

\section{REFERENCES}

1. G.L. Siscoe, Space Plasma Physics (ed: Kenne11, Lanzerotti and Parker), p. 557, 1979.

2. V.C. Liu, Space Sci Rev., 9, 423, 1969.

3. N.H. Stone, U. Samir and K.H. Wright, J. Geophys. Res. in press, 1978.

4. Ya. L. Al'pert, Handbuch der Physik, (ed. S. Flugge) Geophysics III, Part V p. 217, 1976.

5. Ya: L. Al'pert, A.V. Gurevich and L.P. Pitaevsky, Space Physics with Artificial Satellites, Consultant Bureau, 1965.

6. A.V. Gurevich, L.P. Pitaevsky, and V.V. Smirnova, Space Sci. Rev., 9, 805, 1969.

7. S.M. Ca11, Report No. 46, Plasma Laboratory, Columbia Univ., 1969.

8. G. Fournier, O.N.E.R.A., 1971.

9. R. Grabowsky and T. Fischer, Planet. Space Sci., 23, 287, 1975.

10. A.V. Gurevich, and L.P. Pitaevsky, Progr. Aerosp. Sc1., 16(3), 227, 1975.

11. V.C. Liu, Progr. Aerosp. Sci., 16(3) 273, 1975.

12. L.W. Parker, Report, NASA CR-144159, February, 1976.

13. U. Samir, in: Photon and Particle Interactions with Surfaces in Space, edited R.J. Grard, p. 193, D. ReIde1, Hingham, Mass. 1973.

14. U. Samir, M. First, E.J. Maier, and B.E. Troy, J. Atmos. Terr. Phys., 37, 577, 1975.

15. U. Samir, R. Gordon, L.H. Brace and R. Theis, J. Geophys. Res., 84, 513, (1979).

16. U. Samir, L.H. Brace and H.C. Brinton, Geophys. Res. Lett. 6, 101 (1979).

17. U. Samir and N.H. Stone, Acta Astronautica, 1980, In press.

18. S.D. Hester and A.A. Sonin, Phys. Fluids, 13, 641,1970.

19. G. Fournier and D. Pigache, Phys. Fluids, 18, 1443, 1975.

20. U. Samir, N.H. Stone and W.A. Oran, Astrophys, and Space Sci., 31, L1, 1974 . 
21. C.G. Falthammar, Space Sci. Rev., 15, 803, May 1974.

22. L.P. Block, Phys.of Plan. Environments, (Ed. D.J. Williams), vol. 1, 255, 1976.

23. I.M. Podgorny, and Andrijanov, Plan. Space Sci., 26, 99, 1978.

24. U. Samir, E.J. Haier and B.E. Troy, J.Atmos. Terr. Phys. 35, 513, 1973.

25. L.H. Brace, R.F. Theis and A. Dalgarno, Radio Sci., 8(4), 341, 1973.

26. U. Samir and H. Jew, J. Geophys. Res. 77, 6819, 1972.

27. Y.S. Dimant and A.V. Gurevich, Geomagnetism and Aeronomy, 15(2), 183, 1975.

28. L.W. Parker, in: Proc. of the Spacecraft Charging Technology Conference, (ed., C.P. Pike and R.R. Love11), Joint Air Force - NASA Report AFGL-TR77-0051 and NASA TMX-73537, pp. 331-360, February, 1977.

29. U. Samir and Y.J. Kaufman, J. Atmos \& Terrest. Phys. in press, 1980.

30. U. Samir and A.P. Willmore, Planet. Space Sci., 13, 285, 1965.

31. U. Samir and A.P. Willmore, Plan. Space Sci., 14, p. 1131, 1966.

32. C.L. Henderson, and U. Samir, Planet. Space Sci., 15, 1499, 1967.

33. U. Samir and G.I. Wrenn, Planet. Space Sci. 17, 693, 1969.

34. N.J. Miller, J. Geophys. Res. 77(40), 2851, 1972.

35. H. Oya, Plan. Space Sci., 18, 793, 1970.

36. H. Weil and R.G. Yorks, Plan. Space Sci. 18, 801, 1970.

37. D.B. Medved, in: Rarefied Gas Dynamics, edited by L. Trilling and H.Y. Wachman, p. 1525, Academic, New York, 1969.

38. B.E. Troy, Jr., D.B. Medved and U. Samir, J. Astronautical Sci. 18, 173, 1970.

39. U. Samir, and G.L. Wrenn, Planet. Space Sc1. 20, 899, 1972.

40. B.E. Troy, Jr., E.J. Maier, U. Samir, J. Geophys. Res. 80, 993, 1975.

41. J.M. Illiano and L.R.0. Storey, Plan. Space Sci., 22, 873, 1974.

42. A. V. Gurevich, L.P. Pariskaya and L.P. Pitaevsky, Soviet Physics J.E.T.P., $36(2), 274,1973$.

43. H.C. Brinton, L.R. Scott, M.W. Pharo and J.T.C. Coulson, Radio Scie, $8(4), 323,1973$.

44. U. Samir, Y.J. Kaufman, L.H. Brace and H.C. Brinton, J. Geophys. Res. In press, 1980. 